\title{
Exploring the Link Between Amorphous Structure and Crystallization Behavior of Titania Thin Films by Electron-Based Pair Distribution Functions and in-situ TEM
}

John S. Mangum ${ }^{1}$, Lauren M. Garten ${ }^{2,3}$, Valerie Jacobson ${ }^{1,2}$ David S. Ginley ${ }^{2}$ and Brian P. Gorman ${ }^{1}$

1. Department of Metallurgical and Materials Engineering, Colorado School of Mines, Golden, CO 80401, USA

2. National Renewable Energy Laboratory, Golden, Colorado 80401, USA

3. Material Science and Technology Division, U.S. Naval Research Laboratory, Washington, D.C. 20375

Certain structural polymorphs of titanium dioxide $\left(\mathrm{TiO}_{2}\right)$ are known to exhibit photocatalytic ability useful in applications pertaining to water splitting reactions and degradation of organics for water treatment.[1,2] Among these polymorphs, the brookite structure has been shown to possess the greatest photoactivity under a variety of conditions. $[3,4]$ However, brookite is a metastable phase and difficult to synthesize with respect to its polymorphic counterparts, rutile and anatase.

Over the last several decades, many studies have been published detailing a variety of synthesis procedures for producing brookite $\mathrm{TiO}_{2}$, ranging from hydrothermal processes to thin film deposition.[4] Although these report the synthesis methods in detail, there is little to no evidence of exploring the underlying mechanism that stabilizes the brookite polymorph. This work aims to elucidate and understand the fundamental mechanism(s) that lead to not just brookite, but the anatase and rutile structures as well.

Our previous work conducted in this polymorphic landscape demonstrated $\mathrm{TiO}_{2}$ phase selectivity through manipulation of the amorphous thin film precursors prior to crystallization.[5] One parameter revealed to direct crystalline polymorph formation was oxygen pressure $\left(\mathrm{pO}_{2}\right)$ in the chamber during pulsed laser deposition. Changing $\mathrm{pO}_{2}$ was shown to directly influence the stoichiometry of the amorphous precursor film, resulting in higher degrees of oxygen sub-stoichiometry with decreasing $\mathrm{pO}_{2}$. It is possible to precisely target each of the three $\mathrm{TiO}_{2}$ polymorphs through only changes in amorphous precursor stoichiometry, suggesting a major role of defects brought on by oxygen deficiency in directing crystallization pathways.

Removal of oxygen from the amorphous precursor system should result in a reconfiguration of Ti-O octahedra to a lowest energy state. We hypothesize this rearrangement of the amorphous structure to consist of a mixture of corner- and edge-sharing octahedra, with the ratio of the two dependent on stoichiometry. Rutile, brookite, and anatase each have distinct ratios of corner- to edge-sharing octahedra. An amorphous precursor structure exhibiting strong similarity to one of the crystalline phases could promote preferential nucleation and growth of that particular polymorph over the others.

Probing the local atomic structure of amorphous materials can be accomplished through radial distribution function (RDF) analysis. Though typically done with X-ray or neutron scattering techniques, we show in this work that radial distribution functions calculated from transmission electron diffraction patterns are capable of discerning the local atomic configuration of the amorphous titanium oxide precursors discussed above. Figure 1 shows a radial distribution function calculated from a $25 \mathrm{~nm}$ thick amorphous titania film. The first three peaks in the RDF correspond to the Ti-O, Ti-Ti edge-sharing octahedra, and $\mathrm{Ti}-\mathrm{Ti}$ corner-sharing octahedra distances, respectively. By inspecting differences in the intensity ratio of the edge- and corner-sharing octahedra peaks as a function of deposition $\mathrm{pO}_{2}$, we are 
able to gain a better understanding of how the amorphous structure influences crystalline polymorph formation. Furthermore, this electron-based RDF analysis can be conducted in concert with in-situ annealing experiments to directly observe the crystallization process in the TEM. Understanding the atomic structure before and after crystallization as well as the kinetics and microstructure evolution of the crystallization process provides a level of insight necessary for revealing the underlying mechanisms of polymorph formation in the $\mathrm{TiO}_{2}$ system.[6]

\section{References:}

[1] A Fujishima, X Zhang, Comptes Rendus Chimie 9 (2006), 750.

[2] A Wold, Chem. Mater. 5 (1993), 280.

[3] A Paola, M Bellardita, and L Palmisano, Catalysts 3 (2013), 36.

[4] R Bhave, All Theses 66 (2007).

[5] Mangum et al., J. Non-Cryst. Solids 505 (2019), 109.

[6] This work was supported as part of the Center for Next Generation Materials by Design:

Incorporating Metastability, an Energy Frontier Research Center funded by the U.S. Department of Energy, Office of Science, Basic Energy Sciences under Award \# DE-AC36-08GO28308.

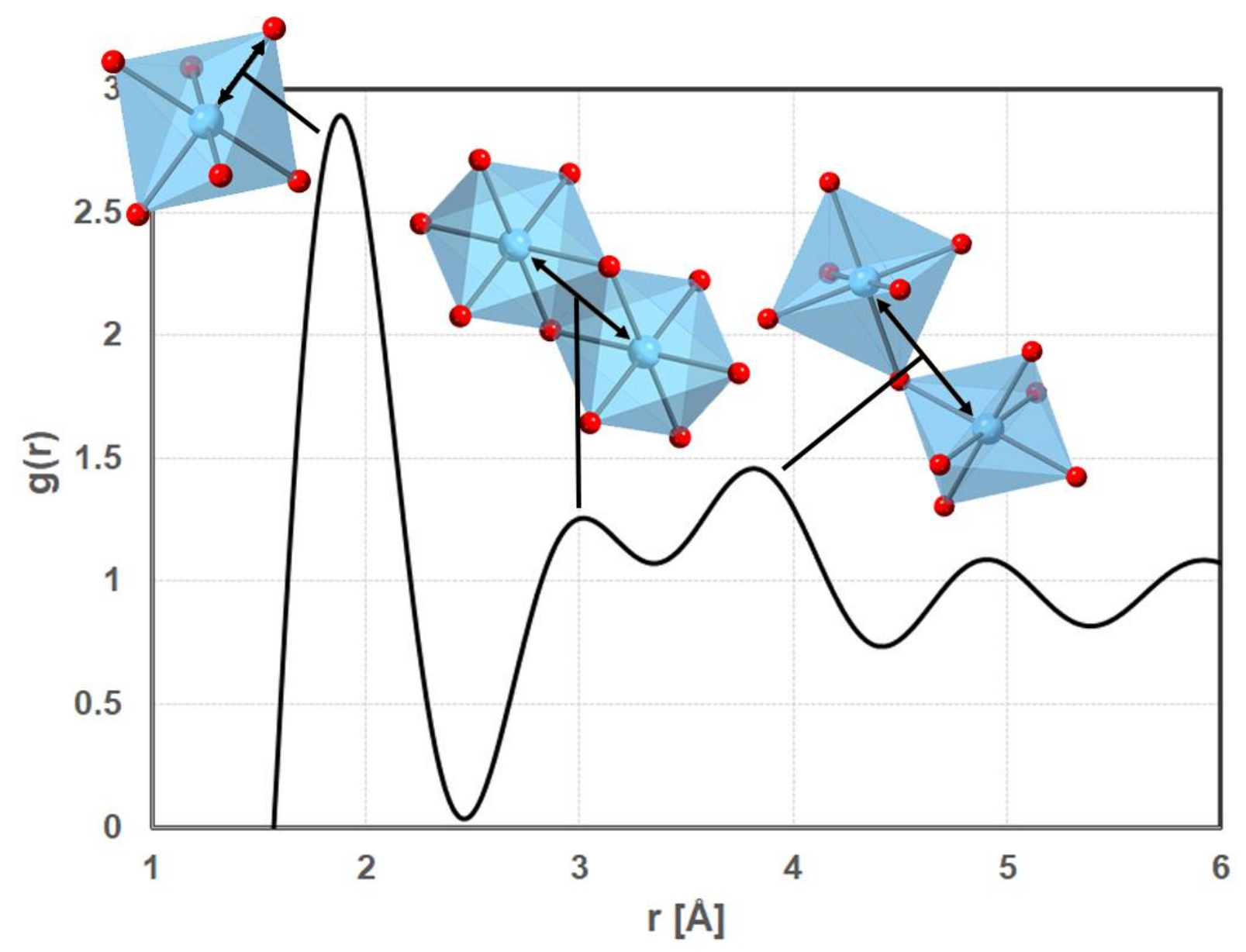

Figure 1. Radial distribution function calculated from a $25 \mathrm{~nm}$ thick amorphous titania film. The first three peaks correspond to Ti-O first coordination shell distance (1.9 $\AA$ ), Ti-Ti edge-sharing octahedra distance $(3 \AA)$, and Ti-Ti corner-sharing octahedra distance $(3.8 \AA)$, respectively. 